\title{
Planar and Vertical Signals Control Cellular Differentiation and Patterning in the Mammalian Cochlea
}

\author{
Mireille Montcouquiol and Matthew W. Kelley \\ Section on Developmental Neuroscience, National Institute on Deafness and Other Communication Disorders-National Institutes of Health, Rockville, \\ Maryland 20850
}

The sensory epithelium of the mammalian cochlea is composed of a regular mosaic of sensory hair cells and nonsensory supporting cells. During development, differentiation occurs in a gradient that progresses along the axis of the cochlea from base to apex. To begin to identify some of the factors that regulate this developmental process, the potential roles of planar and vertical signals were examined during early stages of cochlear development. We demonstrate roles for both underlying mesenchymal cells and adjacent epithelial cells in the differentiation and patterning of the sensory epithelium, and in particular in the development of mechanosensory hair cells. As development proceeds, the requirements for both planar and vertical signals decrease, and development of the sensory epithelium becomes essentially independent from these cues. Finally, we demonstrate that the temporal gradient of cellular differentiation is not dependent on planar signals within the developing sensory epithelium.

Key words: cochlea; mesenchyme; development; hair cell; inner ear; epithelium

\section{Introduction}

In mammals, sounds are perceived by mechanosensory hair cells located within the sensory epithelium of the cochlear duct (the organ of Corti). In contrast with other mechanosensory epithelia, the mature organ of Corti is composed of a regular pattern of specialized cell types, including two types of mechanosensory hair cells as well as at least four types of nonsensory supporting cells. These different cell types are arranged into a repeating mosaic that extends along the length of the cochlear spiral. The presence and appropriate arrangement of many of these cell types have been shown to be essential for the development of normal hearing (Avraham et al., 1995; Weil et al., 1995; Colvin et al., 1996; Chen and Segil, 1999); however, the factors that play a role in the overall development of the sensory epithelium are poorly understood.

In mice, the cochlear duct begins to develop around embryonic day (E) 11.5 as an out-pocketing from the otocyst (Sher, 1971; Lim and Anniko, 1985). By E12.5 this out-pocketing has extended and coiled to form approximately one-half turn. As development proceeds, the duct continues to coil until it reaches a mature length of $\sim 1.5$ turns by E18.5. The results of morphological and molecular studies have suggested that specification and differentiation of individual cell types within the organ of Corti begin between E13.5 and E14 (Xiang and al., 1998; Lanford

Received June 24, 2003; revised Sept. 2, 2003; accepted Sept. 4, 2003.

This research was supported by the Intramural Program at the National Institute of Deafness and Other Communication Disorders and by a research grant from the March of Dimes. We acknowledge Kasey Heintz for technical assistance. We are grateful to Drs. Mark Warchol, Susan Sullivan, Alain Dabdoub, and Inna Belyantseva and to Jason Meyers and Jennifer Jones for critical comments on this manuscript.

Correspondence should be addressed to Mireille Montcouquiol, Section on Developmental Neuroscience, National Institute on Deafness and Other Communication Disorders-National Institutes of Health, 5 Research Court, Rockville, MD 20850. E-mail: montcouq@nided.nih.gov.

Copyright $\odot 2003$ Society for Neuroscience $\quad$ 0270-6474/03/239469-10\$15.00/0 et al., 1999). Cellular differentiation progresses in a gradient that initiates in the mid-basal region of the duct and advances toward the apex. Therefore, it seems possible that signals that are required for the development of the organ of Corti could initiate in this mid-basal region and then progress within the plane of the epithelium toward the apex of the cochlea.

A second possible source of developmental cues could be adjacent cell types such as condensing mesenchymal cells. Data from previous studies have demonstrated that many developing structures, such as the developing neural plate (for review, see Kelly and Melton, 1995; Wessely and De Robertis, 2002), retinal pigmented epithelial cells (Fuhrmann et al., 2000), and early otocyst (Mansour et al., 1993; Maroon et al., 2002), receive instructive cues from closely associated unrelated cell types. The goals of this study were to identify potential sources of signals that are required for the differentiation and patterning of the organ of Corti. The results presented demonstrate for the first time that both planar (referring to signals within the plane of the epithelium) and vertical (referring to signals originating from underlying mesenchyme) cues play a role in the regulation of the development of the organ of Corti.

\section{Materials and Methods}

Cochlear cultures

Pregnant CD-1 mice (Charles River Laboratories, Wilmington, MA) were killed on E12.5 or E13.5, according to the National Institutes of Health Guide for the Care and Use of Laboratory Animals. Embryos were individually staged on the basis of the developmental series presented in Kaufman (1992), and individual cochleas were dissected in cold HBSS (Invitrogen, Carlsbad, CA). After removal of the surrounding cartilage, the anlagae of Reissner's membrane was dissected to expose the developing sensory epithelium. After dissection, the developing sensory epithelium and associated mesenchymal cells were established in an individual MatTek dish (MatTek Corporation, Ashland, MA) as an intact explant 


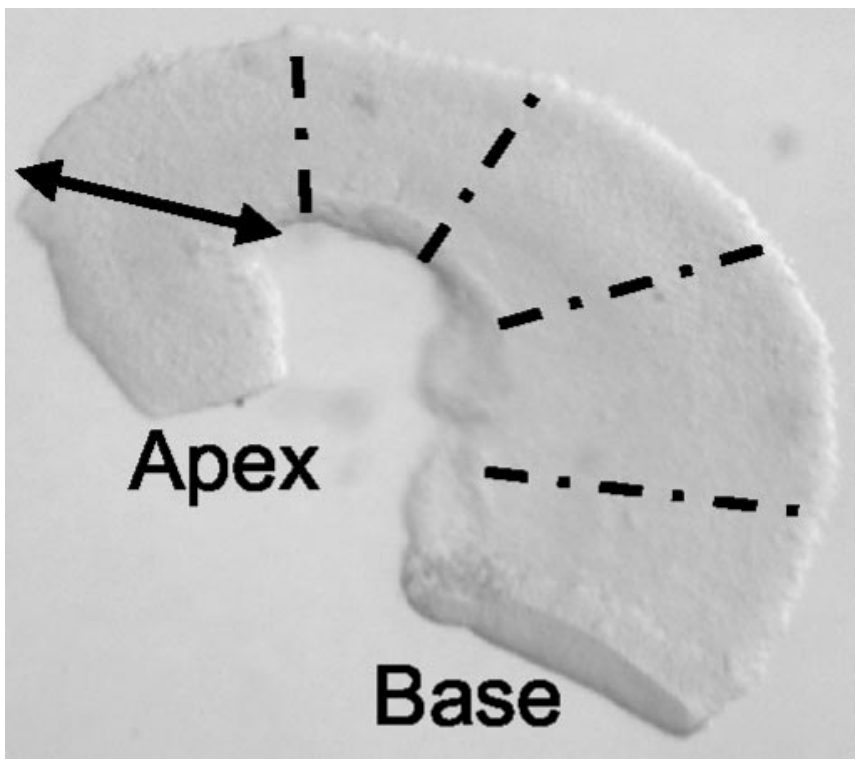

Figure 1. Differential interference contrast image of an intact E13.5 cochlear epithelium after removal of the underlying mesenchymal cells by treatment with thermolysin. Apical (Apex) and basal (Base) ends of the cochlear duct are indicated. The solid line represents the location for separation of the single apical piece. Dashed lines represent subsequent subdivisions to create basal pieces that are of approximately equivalent sizes to the single apical piece.

culture. For some cultures, the apical $20-25 \%$ of the developing epithelium was separated by making a cut perpendicular to the basal-to-apical axis with a sapphire knife as illustrated in Figure 1. Apical pieces were placed in individual MatTek dishes previously coated with poly-L-lysine (Sigma, St. Louis, MO), cell-tak (Becton Dickinson, Mountain View, $\mathrm{CA}$ ), and matrigel (Becton Dickinson). The remaining basal regions either were cultured intact as a single piece or cut into three approximately equal pieces that were then cultured together in a single MatTek dish. The culture medium was composed of DMEM, 10\% fetal bovine serum (FBS), N2 supplement (all from Invitrogen), penicillin, and fungizone. For the experiments in defined medium, the FBS was omitted.

To examine the role of associated mesenchymal cells, after dissection, some cochleas were incubated in a solution of thermolysin in HBSS (250 $\mu \mathrm{g} / \mathrm{ml}$; Sigma) for $20-50 \mathrm{~min}$ in a $35^{\circ} \mathrm{C}$ humidified incubator with $5 \%$ $\mathrm{CO}_{2}$. The organs were then transferred to fresh cold HBSS, and the cochlear epithelium was gently lifted away from the underlying mesenchymal tissue. The resulting sheet of epithelial cells was then either cultured intact or separated into apical and basal pieces as illustrated in Figure 1. After separation from the apical part and as a rule, E12.5 cochlear epithelia were cut into four basal pieces, whereas E13.5 epithelia were cut into five basal pieces, and the individual sheets were allowed to adhere in MatTek dishes. Unless specified, cultures from E12.5 cochleas were maintained for $6 \mathrm{~d}$ in vitro (DIV), and cultures from E13.5 cochlea were maintained for $5 \mathrm{DIV}$. At the end of each experiment, cultures were fixed in $4 \%$ paraformaldehyde (PFA) for $20 \mathrm{~min}$ and processed for immunostaining as described below.

For coculture experiments, E12.5 cochleas were isolated, treated with thermolysin, and isolated into epithelial and mesenchymal components as described. Separated apical epithelial pieces were placed in the center of a MatTek dish, and pieces of mechanically dissociated mesenchymal tissue were arranged around the cochlear pieces in a single $100 \mu \mathrm{l}$ drop of culture medium. The cultures were maintained in the same drop of medium for 6 DIV. Alternatively, E12.5 or E13.5 mesenchymal tissues were mechanically dissociated and placed in culture for 1 or 7 DIV to adhere. After 1 or 7 DIV, E12.5 apices were placed on top of the mesenchymal layer and cultured for an additional 6 DIV in the same drop of culture medium conditioned for 1 or $7 \mathrm{~d}$ by the mesenchymal cells before fixation. Similar experiments were performed using mesenchymal tissues from E13.5 limb buds or cranial region and maintained for 7 DIV before introduction of E12.5 apical pieces.
For some experiments, we collected medium conditioned for $7 \mathrm{~d}$ by E12.5 mesenchymal cells and cultured E12.5 isolated apices in that medium for 6 DIV. Finally, some E12.5 cochleas were treated with thermolysin to disconnect the cochlear epithelium from the underlying mesenchyme, and the resulting epithelial sheets were then carefully placed back on top of the endogenous mesenchymal cells to allow reassociation between the two tissues.

For growth factor experiments, human bone morphogenetic protein 4 (BMP-4) or human recombinant fibroblast growth factor (FGF-10) was added to the culture medium in the presence of $10 \mathrm{ng} / \mathrm{ml}$ heparin and maintained for the duration of the culture (6 DIV).

\section{Immunocytochemistry}

After fixation, samples were rinsed in PBS and incubated in a solution of $5 \%$ normal goat serum in PBS with $0.5 \%$ Tween 20 (PBS-T). The samples were then incubated for $2 \mathrm{hr}$ with primary antibodies that have been shown to label specific cell types within the organ of Corti, as described below. After incubation in the primary antibody, binding was detected by either immunofluorescence or peroxidase immunostaining as described below.

Immunofluorescence. After incubation with the primary antibody, samples were rinsed and incubated with an Alexa Fluor 568-conjugated antibody (Molecular Probes, Eugene, OR), rinsed again, mounted with anti-fade medium (Slowfade Antifade kit; Molecular Probes), and observed through a Nikon E800 microscope or a Zeiss LSM510 Z confocal microscope. Double immunostaining was performed by incubating samples for 90 min with a second primary antibody. After rinsing, samples were incubated with an Alexa Fluor 488-conjugated antibody (Molecular Probes). Some samples were incubated with Alexa Fluor 488-conjugated phalloidin (Molecular Probes).

Peroxidase immunostaining. After incubation in the primary antibody, samples were rinsed and incubated with a biotin-conjugated secondary antibody (Jackson ImmunoResearch, West Grove, PA). Samples were then reacted with diaminobenzidine using an Elite ABC kit (Vector Laboratories, Burlingame, CA) with nickel intensification.

After immunoperoxidase staining with the myosin VI antibody, some cultures were embedded in Immuno-bed (Polyscience, Warrington, PA), a methacrylate-based medium, and sectioned at a thickness of $3 \mu \mathrm{m}$ to examine the cytologic structure of the organ of Corti after 3 DIV.

Cell type-specific antibodies. The following antibodies were used to label specific cell types: myosin VI (gift from Dr. T. Hasson, University of California San Diego, San Diego, CA), myosin VIIa (gift from Dr. C. Petit, Pasteur Institute, Paris, France) or Math1 (Chemicon) to label hair cells, prestin (gift from Dr. B. Kachar, National Institute on Deafness and Other Communication Disorders-National Institutes of Health, Bethesda, MD) to label outer hair cells, and p $75^{\text {ntr }}$ (Chemicon, Temecula, CA) to label pillar cells. The Math1 antibody (Chemicon) was used according to the manufacturer protocol.

For whole-mount immunocytochemistry, E13.5 cochleas were dissected as described, fixed in $4 \%$ PFA overnight at $4^{\circ} \mathrm{C}$, and processed for immunostaining as whole organs. To ensure adequate penetration of antibodies, the incubation time for the primary antibody was increased to an overnight treatment at $4^{\circ} \mathrm{C}$.

\section{Live-dead assay, zVal-Ala-Asp(Ome)-fluoromethyl ketone}

treatment, and N-(3-triethylammoniumpropyl)-4-(4-

(dibutylamino)styryl) pyridiniumdibromide labeling

Live-dead assays were performed on pieces of E13.5 cochlear epithelium after 24 and 48 hr with a Live/Dead Cell-Mediated Cytotoxicity kit (Molecular Probes) according to the manufacturer's protocol.

To inhibit apoptosis, some cultures were maintained in the presence of the pan-caspase inhibitor zVal-Ala-Asp(Ome)-fluoromethyl ketone (zVAD) (Enzyme Systems Products, Livermore, CA) at concentrations of 25 or $12.5 \mu \mathrm{M}$ for the entire time of the culture (Matsui et al., 2002).

To assess the presence of transduction channels, some E13.5 explants were cultured for $7 \mathrm{DIV}$ and then bathed for $5 \mathrm{~min}$ at $37^{\circ} \mathrm{C}$ in $3 \mu \mathrm{M}$ $\mathrm{N}$-(3-triethylammoniumpropyl)-4-(4-(dibutylamino)styryl) pyridiniumdibromide (FM1-43) (Molecular Probes) and finally observed under a confocal microscope within the next $15 \mathrm{~min}$. 


\section{Quantification}

MetaMorph software (Universal Imaging Inc., Media, PA) was used to determine the total number of hair cells per sample (Montcouquiol and Corwin, 2001). To analyze the development of overall cellular pattern, we decided to examine the spatial distribution of hair cells within each explant and to compare this distribution with the pattern observed in vivo. Because the cochlea develops as a spiral, the population of hair cells becomes distributed in an arc that is parabolic in shape and can be described using a polynomial regression (van Ruijven et al., 1999; Malbouisson et al., 2000). To compare the accuracy with which similar patterns developed in explants, the spatial distribution of hair cells from in vivo cochleas at postnatal day 0 were plotted as $X, Y$ coordinates. These data were then used to determine the polynomial equation that most accurately described the parabolic shape of the hair cell distribution. Results indicated that a second order polynomial equation of the form $(y=b+$ $\left.c_{1} x+c_{2} x^{2}\right)$ fit the data with an average correlation coefficient of $0.99 \pm$ 0.005 . Therefore, to assess the accuracy of patterning in explants, the locations of all hair cells within a single explant were plotted as $X, Y$ coordinates, and the resulting data were used to generate a best fit second order polynomial equation and correlation coefficient. These data were used to generate an average correlation coefficient for all explants within a specific treatment group. Significant differences in patterning were determined by $t$ test. Polynomial regression equations were generated only for samples with $>14$ hair cells.

\section{Determination of hair cell density}

To determine the packing density of hair cells in explants maintained in media with or without serum, hair cells were labeled with an anti-myosin VI antibody as described. Then, for each explant a contiguous region of sensory epithelium was outlined using Metamorph. The overall area in square micrometers and the number of hair cells within each region were then determined.

\section{Results \\ Cellular differentiation and patterning are essentially unaffected in entire E13.5 + 5 DIV cochlear epithelia that have been isolated from associated mesenchyme}

To determine whether the factors required for differentiation and patterning of the organ of Corti are contained within the developing epithelium, cochleas were isolated on either E13.5 or E12.5. Developing epithelia were dissected from associated mesenchyme using thermolysin and established in vitro as intact cochlear epithelia. Explants were maintained until the epithelia reached a developmental stage equivalent to E18 in vivo (referred to as E12.5 + 6 DIV and E13.5 + 5 DIV either with $(/+$ mes $)$ or without $(/-$ mes $)$ mesenchyme, unless specified otherwise. After fixation, specific cell types were labeled as described in Materials and Methods. Hair cells and supporting cells developed along the length of the epithelia in cochleas from E13.5 + 5 DIV or E12.5 + 6 DIV (Fig. $2 A, C$ ). In addition, in the basal regions of most E12.5 +6 DIV and E13.5 + 5 DIV explants, hair cells and supporting cells formed a cellular pattern that is characteristic for the organ of Corti, including a single row of inner hair cells, a single row of pillar heads, and three or more rows of outer hair cells (Fig. 2A, C, insets). The presence of functional transduction channels was assayed by treating an intact E13.5 $+5 \mathrm{DIV} /-$ mes epithelium with FM1-43, a fluorescent dye that has been shown to pass through hair cell transduction channels (Gale et al., 2001; Meyers et al., 2003), for $5 \mathrm{~min}$. FM1-43 was internalized by both inner and outer hair cells, demonstrating the presence of functional transduction channels (Fig. $2 \mathrm{~A}$, inset).

A progressive disruption in cellular pattern was observed in more apical regions of E12.5 $+6 \mathrm{DIV} /-$ mes and E13.5 +5 DIV/-mes explants. This disruption included a marked accumulation of outer hair cells and a failure to form discrete rows (Fig. $2 A, C$ ). To determine whether these disruptions in pattern
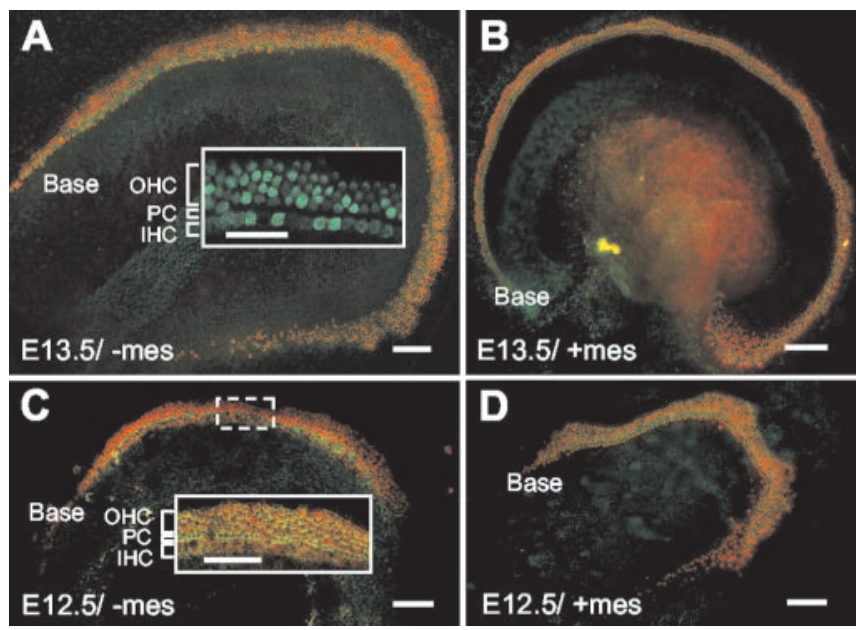

Figure 2. Mesenchymal cells are not required for development of hair cells or cellular patterning. A, Low-magnification image of an intact cochlear epithelium isolated on E13.5 and maintained in the absence of mesenchymal cells (-mes) for 5 DIV (E13.5 + 5 DIV). A band of myosin VI-positive hair cells (red) extends along the entire length of the explant. The inner hair cells are separated from the outer hair cells by a line of pillar cell heads, labeled with the actin marker phalloidin (green). Inset, High-magnification image of the basal region of a living E13.5 + 7 DIV/ - mes intact epithelium. Hair cells have been labeled by rapid uptake of FM1-43, a dye that is thought to enter hair cells through active transduction channels (see Results for details). Note that the hair cells are arranged into a characteristic pattern of a single row of inner hair cells (IHCS) and three rows of outer hair cells (OHCS). The row of pillar cell heads (PCS) located between the row of inner hair cells and first row of outer hair cells is devoid of staining. $B$, Low-magnification view of an intact E13.5 + 5 DIV/-mes cochlear epithelium. A characteristic pattern of inner and outer hair cells is present. Hair cells and pillar cells are labeled as in A. C, Lowmagnification image of an intactE12.5 + 6DIV/-mes cochlear epithelium. Hair cells and pillar cells are labeled as in $A$. A characteristic cellular pattern is present in the basal region of the explant (Base), but supernumerary outer hair cells are present in the apex. Inset, High-magnification view of the boxed region illustrating the cellular pattern of the organ of Corti, including one row of IHCs separated from the three or more rows of $0 \mathrm{HCs}$ by the PCS.D, Low-magnification view of an intactE12.5 + 6DIV cochlear duct maintained with mesenchymal cells (+mes). Hair cells and pillar cells are labeled as in $A$. Note the similar development of supernumerary outer hair cells in the apical region of the explant. Scale bars: $A-D, 100 \mu \mathrm{m} ; A, C$, insets, $50 \mu \mathrm{m}$.

were a result of the removal of underlying mesenchymal cells, similar explants were established with associated mesenchyme still attached (without thermolysin treatment). Analysis of these explants demonstrated similar patterning defects in the apical regions of the cochlear duct (Fig. $2 B, D$ ), suggesting that these patterning defects are a result of explantation rather than removal of underlying mesenchymal cells. Similar results have also been reported for apical regions of intact embryonic cochlear explant cultures and, to a lesser extent, in apical regions of the cochlea in vivo (Lavigne-Rebillard and Pujol, 1987; Abdouh et al., 1994). It has been suggested recently that convergent extension may play a role in cellular patterning within the cochlea (Chen et al., 2002; Montcouquiol et al., 2003). Therefore defects in convergent extension that may occur as a result of explantation could explain these results.

\section{Cellular differentiation and patterning are not disrupted in} basal pieces of E13.5 + $5 \mathrm{DIV} /$ - mes cochlear epithelia Previous studies have demonstrated that cellular differentiation in the organ of Corti occurs in a wave that begins in the mid-basal region of the cochlea and extends along the basal-to-apical axis. To determine whether this gradient plays a role in cellular differentiation or patterning, cochlear epithelia were isolated from underlying mesenchymal tissue on E12.5 or E13.5 and separated into apical and basal pieces as described in Materials and Meth- 
Table 1. Development and alignment of hair cells in apical (Apex) and basal (Base) cochlear pieces maintained with or without mesenchyme

\begin{tabular}{|c|c|c|c|c|}
\hline \multirow[b]{2}{*}{ Conditions } & \multicolumn{2}{|l|}{ Apex } & \multicolumn{2}{|l|}{ Base } \\
\hline & $R^{2}$ value & $\begin{array}{l}\text { Number of hair } \\
\text { cells per piece }\end{array}$ & $R^{2}$ value & $\begin{array}{l}\text { Number of hair } \\
\text { cells per piece }\end{array}$ \\
\hline In vivo $(\mathrm{PO})$ & $\begin{array}{c}0.98 \pm 0.006 \\
n=3\end{array}$ & ND & $\begin{aligned} 0.99 & \pm 0.004 \\
n & =3\end{aligned}$ & ND \\
\hline E13.5 + 5 DIV/-mes & $\begin{array}{c}0.79 \pm 0.03^{*} \\
n=20 \\
p=0.01\end{array}$ & $\begin{array}{c}99.8 \pm 12.9 \\
n=20\end{array}$ & $\begin{aligned} 0.82 & \pm 0.02 * \\
n & =21 \\
p & =0.001\end{aligned}$ & $\begin{array}{r}109 \pm 12 \\
n=21\end{array}$ \\
\hline E12.5 + 6 DIV/-mes & ND & $\begin{array}{l}7 \pm 1.5^{*} \\
n=10 \\
p=0.001\end{array}$ & $\begin{array}{c}0.77 \pm 0.08^{*} \\
n=12 \\
p=0.03\end{array}$ & $\begin{array}{c}62 \pm 9.8^{*} \\
n=12 \\
p=0.01\end{array}$ \\
\hline $\mathrm{E} 12.5+6 \mathrm{DIV} /+$ mes & $\begin{aligned} 0.92 & \pm 0.02^{*} \\
n & =3 \\
p & =0.01\end{aligned}$ & $\begin{array}{c}169 \pm 46 \\
n=3\end{array}$ & ND & ND \\
\hline $\begin{array}{l}\text { E12.5 + } 6 \text { DIV reassociated } \\
\text { with endogenous mesenchyme }\end{array}$ & ND & $\begin{array}{l}>100 \text { in each } \\
n=4\end{array}$ & ND & ND \\
\hline
\end{tabular}

$R^{2}$ values indicate the average overall correlation coefficient for alignment for hair cells in each condition \pm SEM. The $R^{2}$ values for E13.5 +5 DIV $/-$ mes apex and base and E12.5 +6 DIV $/-$ mes base all differed significantly from the $R^{2}$ values for in vivo (indicated by *), and the $p$ values for each are included in the table. Number of hair cells per piece indicates the average number of hair cells per piece for each condition \pm SEM. The values for number of hair cells for apex E12.5 + 6 DIV/ - mes represents the mean number of hair cells for those explants in which hair cells developed. The value for number of hair cells in E12.5 + 6 DIV $/$ - mes differed significantly from E13.5 + 5 DIV/ - mes apex and base and E12.5 + 6 DIV/ - mes base and E12.5 + 6 DIV/ + mes (p for all comparisons was 0.001 ). Number of hair cells in E12.5 + 6 DIV/ - mes base was also significantly different from E13.5 + 5 DIV/ - mes. ND, Not determined.

ods (Fig. 1). Because the resulting apical piece represented between 20 and $25 \%$ of the total length of the cochlear duct and to ensure that any effects on cochlear development were not an effect of reduced size, the remaining basal piece was separated into four or five pieces (depending on age), each of which was approximately equal in size to the single apical piece. To confirm that all basal pieces were approximately equal in size, average areas were determined for a subset of pieces before culture. We found no significant difference in the average areas between basal pieces from E13.5 $\left[66,685 \pm 3263 \mu \mathrm{m}^{2}\right.$ (average \pm SEM); $\left.\left.n=6\right)\right]$ and E12.5 $\left(68,228 \pm 4680 \mu \mathrm{m}^{2} ; n=8\right)$.

Hair cells developed in $100 \%$ of basal pieces cultured in the absence of mesenchyme. The average number of hair cells was $109 \pm$ 12 in E13.5 + $5 \mathrm{DIV} /$ - mes basal pieces $(n=21)$ and $62 \pm 9.8$ in E12.5 + 6 DIV/-mes basal pieces $(n=20)$ (Table 1$)$. Because the average size of each basal piece was the same for E12.5 + 6 DIV/ - mes and E13.5 + 5 DIV/-mes cochleas, this result demonstrates that, in the absence of mesenchyme, a significantly greater number of hair cells will develop in basal pieces isolated at E13.5 + 5 DIV.

Development of the characteristic cellular mosaic of the organ of Corti, including a single row of inner hair cells, a row of pillar heads, and three rows of outer hair cells, was clearly present in most E13.5 + $5 \mathrm{DIV} /-$ mes basal pieces (Fig. $3 \mathrm{~A}, \mathrm{C}$ ). In contrast, hair cells in basal pieces of E12.5 + $6 \mathrm{DIV} /$ - mes epithelia developed only a rudimentary cellular pattern in which hair cells were aligned to form an arc, but specific rows of inner and outer hair cells were not obvious (Fig. 3B).

To confirm that the gap observed between the row of inner hair cells and the first row of outer hair cells in E13.5 + 5 DIV/ - mes basal pieces was composed of pillar cells, explants were labeled with anti-p75 ${ }^{\text {ntr }}$ (a pillar cell marker) and anti-myosin VIIa (a hair cell marker). The expression of $\mathrm{p} 75^{\mathrm{ntr}}$ in the row of cells located between the single row of inner hair cells and first row of outer hair cells confirmed the presence of pillar cells (Fig. 3D). Similar expression of $\mathrm{p} 75^{\mathrm{ntr}}$ was observed in E12.5 $+6 \mathrm{DIV} /$ - mes explants despite the fact that hair cells were not organized into regular rows (data not shown).

To determine whether specific types of hair cells developed in basal epithelial pieces, the expression of prestin, a specific marker for outer hair cells (Zheng et al., 2000), was determined in E13.5 $+5 \mathrm{DIV} /-$ mes basal explants. Prestin was expressed in hair cells considered to be outer hair cells on the basis of their position in the mosaic (Fig. 3E) but not in hair cells located in a position consistent with the row of inner hair cells (Fig. 3E, arrows). Analysis of apical and basal samples labeled with phalloidin also demonstrated the development of stereociliary bundles on many hair cells, indicating that the establishment of luminal-basal cellular polarity was not disrupted in the absence of mesenchymal cues (Fig. 3F, arrows).

To be able to compare the development of cellular pattern between explants that were established and maintained under different conditions, the relative spatial arrangements of hair cells within individual explants were plotted in an $X, Y$ coordinate plane, as explained in Materials and Methods, and the corresponding correlation coefficient $\left(R^{2}\right.$ value) was considered to be a measure of the overall quality of the spatial arrangement of hair cells within each explant. For E13.5 + 5 DIV/-mes basal pieces, the average correlation coefficient was $R^{2}=0.81 \pm 0.02$, whereas for E12.5 + $6 \mathrm{DIV} /$ - mes basal explants the average correlation coefficient was $0.76 \pm 0.08$ (Table 1 ). Both of these values were significantly different from the value for hair cells in the organ of Corti in vivo $\left(R^{2}=0.99 \pm 0.01 ; p=0.01\right)$, indicating that the overall alignment of hair cells was disrupted in basal pieces.

\section{The cues required for hair cell differentiation and cellular patterning are essentially endogenous by E13.5}

The results of our previous experiments suggested that the factors required for cellular differentiation and patterning are endogenous to the cochlear epithelium by E13.5. It was possible, however, that undefined factors within the culture media could also play a role in cellular differentiation and patterning. To examine this possibility, basal pieces of epithelia were established from E13.5 cochleas as described but were maintained in defined media for $5 \mathrm{~d}$ in vitro. Analysis of these explants indicated that they contained an identifiable cellular mosaic that included inner and outer hair cells and pillar cells (Fig. 3G). In many cases, however, two or more rows of inner hair cells (Fig. $3 G$, arrows) and more than three rows of outer hair cells were clearly present. An explanation for this disruption in the typical cellular pattern might be related to the fact that explants maintained in defined media did not spread on the substrate to the same extent as comparable cultures maintained in media with serum. As a result, inner and outer hair cells were more tightly packed, partially disrupting the characteristic cellular pattern. To test this hypothesis, the average 

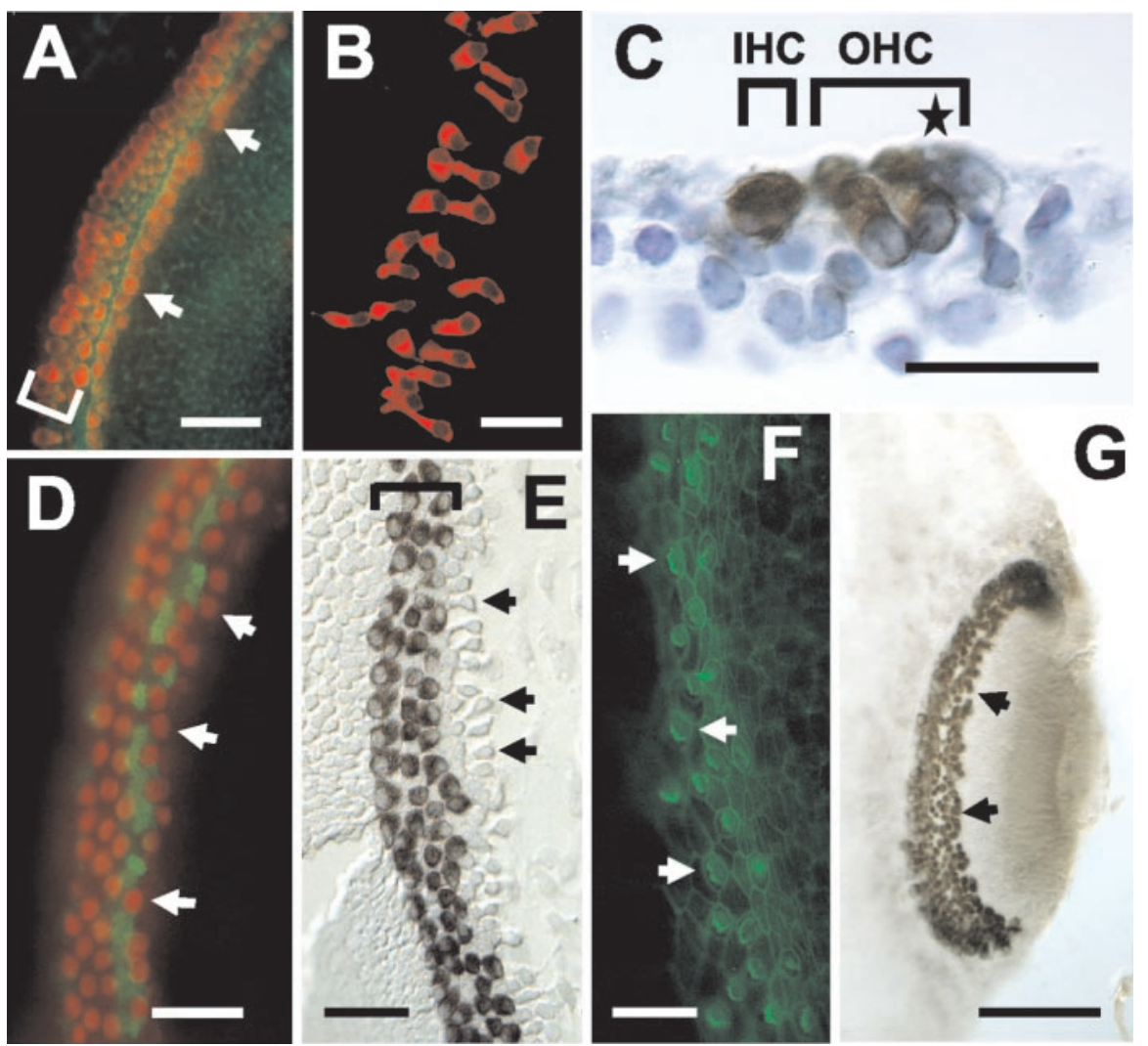

Figure 3. Hair cell differentiation and alignment in basal and apical pieces of cochlear epithelium isolated from mesenchyme and other regions of the cochlea on E13.5 or E12.5. A, Basal cochlear epithelial piece from an E13.5 + 5 DIV/-mes explant. Hair cells (labeled with anti-myosin VI in red) are arranged in a single row of inner hair cells (arrows) and three rows of outer hair cells (brackets) separated by a single row of pillar heads (labeled with phalloidin in green). B, Basal epithelial piece from an E12.5 +6 DIV/- mes explant. Hair cells (labeled with anti-myosin VI in red) are arranged in a line, but specific rows of inner and outer hair cells have not developed. C, Cross section of the sensory epithelium in a basalE13.5 + 3 DIV/-mes explant. A single inner hair cell (IHC) and two outer hair cells (OHCs) are labeled with anti-myosin VI. A third outer hair cell (star) is also present but is stained less intensively. D, Basal epithelial piece from an E13.5 + 5DIV/- mes explant. The pillar cell marker p75 ${ }^{\mathrm{ntr}}$ (green) is expressed in the region between the inner (arrows) and outer hair cells (stained in red with anti-myosin VIla). E, Basal epithelial piece from an E13.5 $+5 \mathrm{DIV} /$ - mes explant. Prestin, a specific marker for outer hair cells, is expressed in outer hair cells (bracket) but not in inner hair cells (arrowheads). F, Apical epithelial piece from an E13.5 + 5 DIV/ - mes explant labeled with phalloidin. Stereociliary bundles (arrows) are intensely labeled with phalloidin (green) and have a "(" morphology that is characteristic of cochlear hair cells. In addition, the presence of stereociliary bundles indicates that the hair cells have become polarized along the luminal-basal axis. $G$, Basal epithelial piece from E13.5 + 5 DIV/-mes explant. An arc of hair cells (labeled with anti-myosin VI) extends along the entire length of the piece, and a disrupted but recognizable cellular pattern is present. The apparent increase in the total number of hair cells by comparison with basal pieces maintained in medium with serum is probably caused by a lack of spreading of the epithelial piece. Scale bars: $A, B, 50 \mu \mathrm{m} ;(-F, 25 \mu \mathrm{m} ; G, 100 \mu \mathrm{m}$.

packing density for hair cells was determined in E13.5 + 5 DIV/mes basal explants maintained either with or without serum. In explants maintained with serum, the average packing density of hair cells was $0.323 \pm 0.168$ per $100 \mu \mathrm{m}^{2}$. By comparison, in explants maintained without serum, the hair cell packing density increased to $1.350 \pm 0.253$ cells per $100 \mu \mathrm{m}^{2}$, a significant increase $(p=0.01)$

Cellular differentiation is disrupted in E12.5 + 6 DIV - mes apical epithelial pieces but not in E13.5 + 5 DIV/-mes apical pieces

To determine whether developmental signals progress into the apical region of the duct over time, apical epithelial pieces were isolated from E12.5 or E13.5 cochleas as described and maintained and analyzed as in the previous section. Analysis of apical pieces from E13.5 + $5 \mathrm{DIV} /$ - mes indicated that hair cells were present in $100 \%$ of the samples and that the average number of

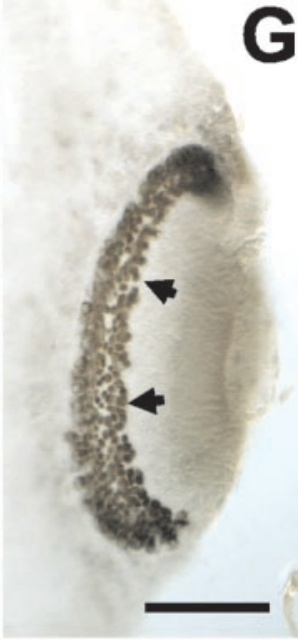

hair cells per apical piece was $99.8 \pm 12.9$ (Fig. $4 A$, Table 1). In most cases, hair cells were arranged in an arc that was comparable with the arrangement of hair cells in basal pieces from either E13.5 + 5 DIV/ - mes or E12.5 + 6 DIV/-mes (Table 1); in several cases a characteristic cellular pattern of inner and outer hair cells could be identified, but in the majority of samples an obvious separation between hair cell types was not evident.

In contrast to apical pieces from E13.5 + $5 \mathrm{DIV} /-$ mes, hair cells developed in only $57 \%$ of apical pieces from E12.5 + 6 DIV/-mes. In addition, in those pieces that did develop hair cells, the average number was only of $7 \pm 1.5$ hair cells per piece, and the overall cellular pattern appeared to be disrupted (Fig. 4 B). The small number of hair cells in these pieces prevented the calculation of correlation coefficients for spatial arrangements.

To rule out the possibility that the difference in number of hair cells in E12.5 + $6 \mathrm{DIV} /-$ mes apical pieces compared with $\mathrm{E} 13.5+5 \mathrm{DIV} /-$ mes pieces was the result of an induction of apoptotic cell death, apical pieces from E12.5 + $6 \mathrm{DIV} /-$ mes cochleas were treated with the pan-caspase inhibitor zVAD. Treatment with zVAD had no apparent effect on the number of cells that developed as hair cells at either 12.5 or $25 \mu \mathrm{M}$ (data not shown). Moreover, to examine any potential toxic effects after the thermolysin treatment, E13.5 + 5 DIV/-mes apical and basal pieces were analyzed using a live-dead assay after 24 and $48 \mathrm{hr}$ in vitro. Limited numbers of dead cells were observed at either time point (data not shown). On the basis of these findings, it seems unlikely that the decrease in the number of hair cells in apical and basal pieces from E12.5 + 6 DIV/-mes is a result of either apoptotic or necrotic cell death.

To begin to identify the specific developmental events that are disrupted in isolated apical epithelia, expression of the transcription factor Math1 was determined in intact cochlear epithelia and in isolated basal and apical pieces from E12.5 + $6 \mathrm{DIV} /-$ mes. Math1 is expressed in the developing sensory epithelium beginning around E13.5 (Bermingham et al., 1999; Lanford et al., 2000) and has been shown to be necessary for hair cell development (Bermingham et al., 1999). A band of Math1-positive cells was present along the length of an E12.5 + $6 \mathrm{DIV} /-$ mes explant (Fig. $5 A, D$ ). In contrast, only a limited number of Math1-positive cells were observed in isolated apical pieces of E12.5 + $6 \mathrm{DIV} /$ - mes (Fig. $5 B, C, E$ ). These results suggest that the induction or maintenance of Math1 is markedly decreased in apical pieces from E12.5 + $6 \mathrm{DIV} /-$ mes.

\section{Mesenchymal cells are sufficient for cellular differentiation and patterning in E12.5 + 6 DIV apical pieces}

To determine whether the mesenchymal cells located adjacent to the basement membrane of the cochlear duct could provide cues 


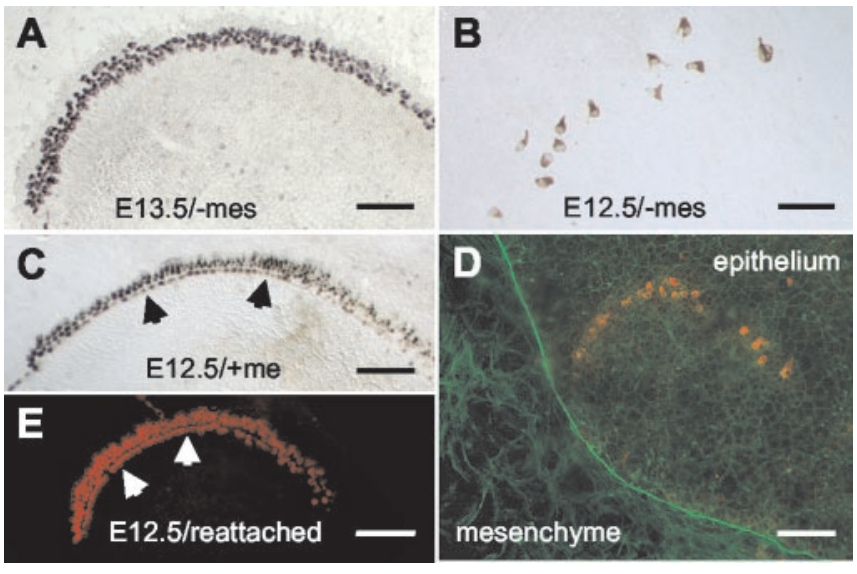

Figure 4. Associated mesenchymal cells are instructive for hair cell differentiation and patterning. A, Apical epithelial piece from an E13.5 + 5 DIV/-mes. An arc of hair cells (labeled with anti-myosin VI in all panels) extends along the length of the explant. $B$, Apical epithelial piece from an E12.5 + 6DIV/ - mes explant. The number of hair cells is significantly reduced by comparison with an apical piece from E13.5 + 5 DIV/ - mes (Table 1), and no cellular pattern is evident. C, Apical piece from an E12.5 + 6DIV/ + mes explant. By comparison with apical pieces cultured without mesenchyme $(B)$, there is a significant increase in the number of hair cells (Table 1) and a marked improvement in the level of cellular patterning (rows), including the formation of specific rows of hair cells and a row of pillar heads. D, Apical epithelial piece from an E12.5 + 6 DIV/-mes explant that was maintained on top of a monolayer of mesenchymal cells (visualized by labeling with phalloidin in green) that were isolated from an E13.5 cochlea and maintained for 7 DIV before introduction of the apical epithelial piece. The number and pattern of hair cells (red) appear similar to apical epithelial pieces maintained without mesenchymal cells. E, Apical epithelial piece from an E12.5 + 6 DIV explant that was reassociated with its endogenous underlying mesenchyme (see Results for details). There is a significant increase in the number of hair cells, and a characteristic pattern of hair cells and supporting cells is present. Scale bars, $100 \mu \mathrm{m}$.

for differentiation and patterning, apical pieces were separated from E12.5 cochleas as described, but associated mesenchymal cells were not removed. The presence of mesenchymal cells led to a significant increase in the number of cells that developed as hair cells and to a marked improvement in overall cellular alignment (Fig. 4C, Table 1). The number of E12.5 $+6 \mathrm{DIV} /+$ mes apical explants with hair cells was 100\% (by comparison with 57\% for E12.5 + 6 DIV/-mes apical explants), and the average number of hair cells per piece was $169 \pm 46$, a significant increase from the $7 \pm 1.5$ hair cells per piece that was observed in apical epithelia without mesenchyme (Table 1 ). In addition, the average correlation coefficient for hair cell alignment in these pieces was $0.92 \pm$ 0.02 (Table 1), a significant increase from the value in E12.5 +6 DIV/ - mes explants, although still significantly different from in vivo (Table 1). Finally, hair cells were clearly arranged into a single row of inner hair cells, a space for the pillar heads and two to three rows of outer hair cells (Fig. 4C).

The results of the previous experiments suggested that cues arising from mesenchymal cells play a key role in cochlear development. To begin to determine the nature of these cues, apical epithelial pieces and mesenchymal cells from the same E12.5 cochlear duct were placed in separate, nonoverlapping regions of a single MatTek dish, and the two groups of cells were cocultured in a single drop $(100 \mu \mathrm{l})$ of culture media for the duration of the experiment (6 DIV). Analysis of the number of hair cells that developed in these E12.5 + $6 \mathrm{DIV} /-$ mes apical epithelial pieces indicated no significant change in comparison with E12.5 +6 DIV/-mes apical pieces maintained in the complete absence of mesenchyme (data not shown). Similarly, the number of hair cells did not increase in apical E12.5 + 6 DIV/-mes pieces cul-
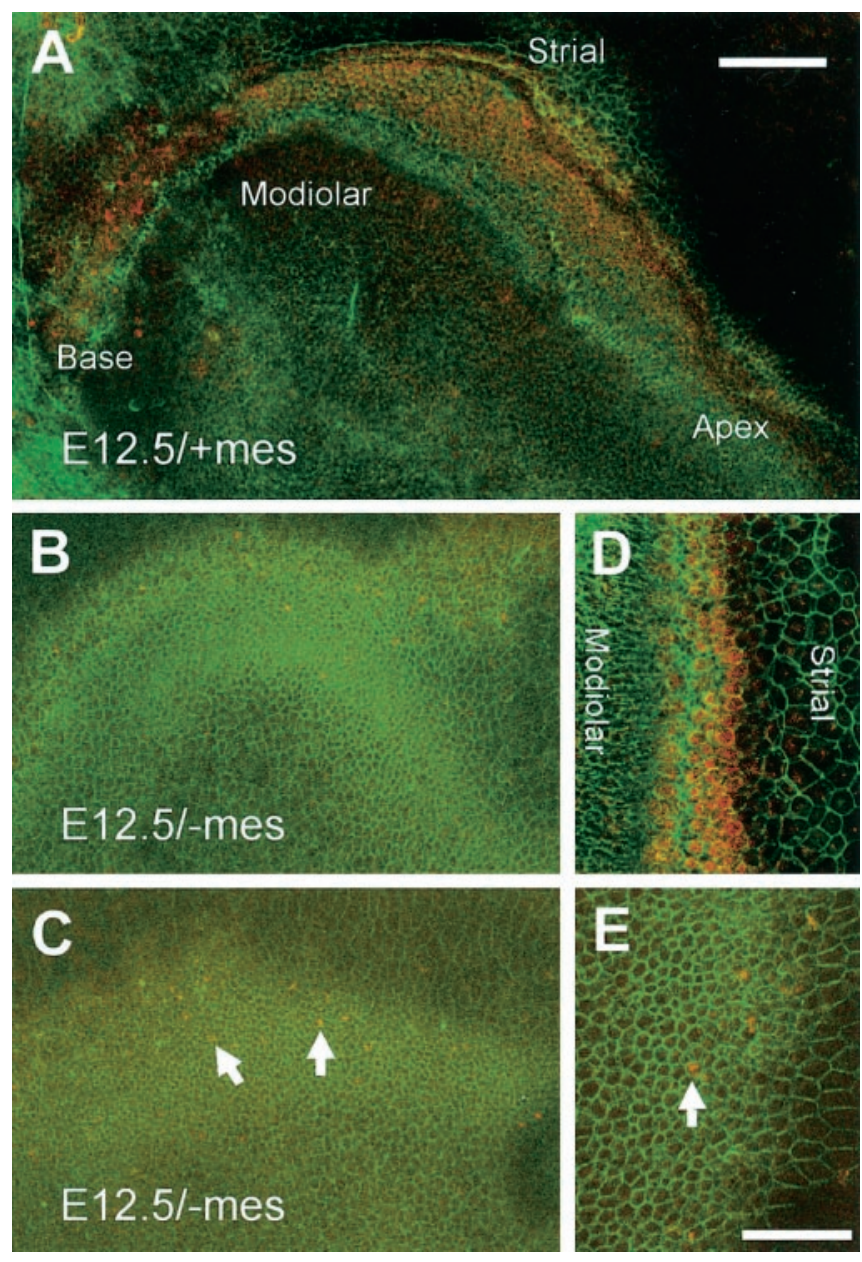

Figure 5. Expression of Math1 in E12.5 + 6 DIV epithelia. A, Math1-positive cells (red) are present in an arcthat extends along the length of the explant in an intact E12.5 + 6 DIV/+ mes. To aid in visualization of the sensory epithelium, filamentous actin has been labeled in green. Orientation of the explant is as indicated. B, C, Isolated E12.5 + 6 DIV/- mes apices labeled and oriented as in $A$. The region of high cell density in each explant corresponds with the area that would normally develop as the sensory epithelium. Only a few Math1-positive cells were detected in either sample (C, arrows). D, High-magnification image of the sensory epithelium from an intact E12.5 +6 DIV explant with mesencyme labeled as in A. Note the single row of Math1-positive inner hair cells and the three rows of Math1-positive outer hair cells. E, Highmagnification image of the high cell density region from an isolated E12.5 + 6 DIV apex. Few Math1-positive cells are present (E, arrow). Scale bar: (in $A) A-C$, $100 \mu \mathrm{m}$; (in $E) D, E, 50 \mu \mathrm{m}$.

tured in media that had been conditioned for 7 DIV by E12.5 cochlear mesenchymal cells (data not shown).

To address the possibility that mesenchymal cells provide nonspecific trophic support for cochlear epithelial development, individual E12.5 apical epithelial pieces without cochlear mesenchyme were placed onto monolayers of embryonic mesenchymal cells that had been isolated from either the developing limb bud or the cranial region on E13.5. In addition, E12.5 apical epithelial pieces without cochlear mesenchyme were also established on top of layers of mesenchymal cells from E12.5 or E13.5 cochleas that had been maintained in vitro for 1 or $7 \mathrm{~d}$ before the introduction of E12.5 apical pieces (Fig. 4D). No obvious increase in the number of hair cells was observed in E12.5 + 5 DIV apical pieces under any of these experimental conditions. These results suggest that nonspecific diffusible factors or cell-cell interactions between mesenchymal cells and cochlear epithelia are not sufficient to induce hair cell development.

The results of the previous experiments suggested that specific 
cues that influence cellular differentiation and patterning in the cochlea may be restricted to the mesenchymal cells located adjacent to the cochlear epithelia. To examine this possibility, apical E12.5 epithelial pieces were treated with thermolysin and dissected away from their associated mesenchyme as described. The epithelial pieces were then placed back on top of their endogenous associated mesenchymal tissue, allowing contact between epithelial and mesenchymal cells from the same region of the cochlear duct to be reestablished. Analysis of hair cell development in these E12.5 + 6 DIV apical pieces indicated a significant increase in the number of cells that developed as hair cells in comparison with apical epithelial pieces isolated from mesenchyme. In the pieces analyzed $(n=4)$, there was a minimum of 100 hair cells per piece and a cellular pattern similar to that in E12.5 + 6 DIV apices isolated and cultured without removal of the endogenous mesenchyme (Fig. 4, compare $C, E$ ).

\section{BMP-4 and FGF10 do not induce hair cells in isolated apical pieces}

The previous results strongly suggest that mesenchymal cells located adjacent to the developing sensory epithelium produce a signal or signals that are required for the expression of Math1 and the formation of the sensory epithelium. The results of previous studies have identified several secreted factors that are specifically expressed in mesenchymal cells that could act as inducing factors. These include BMP-4 and FGF-10 (Liu et al., 2003; Wright and Mansour, 2003). To determine whether either of these factors could act to positively influence sensory epithelium development, isolated E12.5 apical explants were established as described and maintained in media containing either BMP-4 $(200 \mathrm{ng} / \mathrm{ml})$ or FGF-10 (300 ng/ml) for $6 \mathrm{~d}$. The presence of either BMP-4 or FGF-10 failed to increase the number of cells that developed as hair cells (data not shown), suggesting that these factors are not sufficient for the development of the sensory epithelium.

\section{Myosin VI-positive cells are present in the apical region of the organ of Corti at E13.5}

The observation that hair cell differentiation occurred in the apical region of intact E12.5 + 6 DIV/-mes cochlear explants, but was disrupted in the same region of the epithelium if the apical region was isolated from the basal region of the cochlea, suggested that at least some of the signals required for cellular differentiation progressed from the basal to apical regions of the cochlea. Moreover, because the number of hair cells that differentiated was significantly increased in apical pieces from E13.5 + 5 DIV/ - mes by comparison with E12.5 + 6 DIV/ - mes, it seemed likely that some of these signals move into the apical region of the cochlea between E12.5 and E13.5. The results of previous studies had reported the expression of myosin VI protein, an early marker of hair cell development, in the inner ear as early as E13.5 but had not determined its specific spatial pattern of expression in the developing cochlear duct (Xiang et al., 1998). We therefore examined the expression of myosin VI in whole mounts of in vivo cochleas at E12.5 and E13.5 to determine the progress of differentiating hair cells between the two time points.

Myosin VI was not detected at any position along the basalto-apical axis of the cochlea at E12.5, but myosin VI-positive cells were observed in the developing saccule at this time (data not shown). In contrast, by E13.5, a single line of myosin VI-positive cells extended along the basal-most $75 \%$ of the length of the developing cochlea (Fig. 6A,B). Although the line of expression of myosin VI did not extend to the extreme apex of the cochlea at E13.5, it seems likely that some myosin VI-positive cells, or at

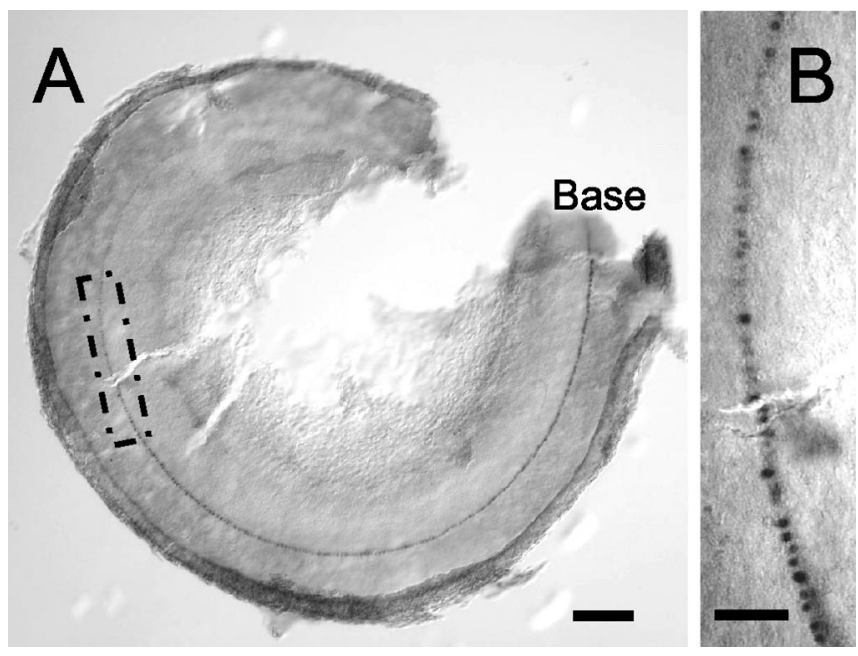

Figure 6. Differentiating hair cells are present in the cochlear epithelium at E13.5 in vivo. A, Whole mount of the entire cochlear duct at E13.5 in vivo. A single line of myosin VI-positive cells extends along the basal $75 \%$ of the cochlea. $B$, High-magnification image of the boxed region in $A$, illustrating the single line of myosin VI-positive presumptive inner hair cells. Scale bars: $A$, $100 \mu \mathrm{m} ; B, 25 \mu \mathrm{m}$.

least some of the signals required to initiate myosin VI expression, were included in apical pieces established at this time point.

\section{The gradient of cellular differentiation is maintained in isolated apical pieces of E12.5 cochleas}

As discussed, cellular differentiation begins in the basal part of the cochlea and then progresses in a wave that extends along the basal-to-apical axis. However, because the terminal mitoses of the progenitor cells that will develop as inner and outer hair cells occurs in an apical-to-basal gradient (Ruben, 1967), cells located in the apical region of the cochlea apparently exist in an undifferentiated state for some period of time. On the basis of results presented here, it seemed possible that planar signals could play a role in coordinating this wave of differentiation. To examine the potential role of planar signals in this event, E12.5 cochleas with associated mesenchymal cells were separated into single apical (approximately top 20-25\%) and single basal (approximately bottom 75-80\%) pieces and established in vitro. Individual samples (each composed of the separated apical and basal part of one cochlea) were fixed after 1, 2, 3, or 4 DIV $($ E12.5 +1 DIV,+2 DIV, +3 DIV, +4 DIV), and expression of myosin VI was determined by immunostaining. In E12.5 + 1 DIV, no myosin VI-positive cells were present in apical or basal pieces (Fig. 7A). After 2 DIV, however, a small number of myosin VI-positive cells could be identified in the mid-basal region of basal pieces (Fig. $7 B$, arrowheads). In contrast, no myosin VI-positive cells were present in any region of apical pieces after 2 DIV. Myosin VIpositive cells were observed along the entire length of basal pieces at 3 DIV (Fig. 7C). Myosin VI-positive cells were also present in apical pieces at $3 \mathrm{DIV}$, and the cells that stained most intensely were concentrated near the basal-most region of the apical piece (Fig. 7C, inset). Finally, at $4 \mathrm{DIV}$, myosin VI-positive cells were observed along the length of both basal and apical pieces (Fig. 7D).

\section{Discussion}

\section{Cellular differentiation and patterning in the cochlea is} autonomous by E12.5

The goals of this study were to begin to identify the sources of cues that are necessary for cellular differentiation and patterning 
in the embryonic organ of Corti and to determine at what stage these events became autonomous to the developing epithelium. Remarkably, most of the cues required for cellular differentiation and patterning appear to be present within the basal region of the developing epithelium by E12.5 and throughout the basal-toapical axis by E13.5. Specific cell types, including inner and outer hair cells and supporting cells, developed along the length of the explant in intact cochlear epithelia from either E12.5 + 6 DIV/ - mes or E13.5 + 5 DIV/-mes. Moreover, the characteristic mosaic pattern, including a single row of inner hair cells, a row of pillar heads, and three or more rows of outer hair cells, was observed in at least the basal region of all samples. Similar results were obtained for E13.5 + 5 DIV/-mes explants maintained in defined media, demonstrating that soluble factors produced outside the epithelium are not required for cellular differentiation and patterning. These results strongly suggest that cues derived from external sources, such as the developing spiral ganglion or spiral vessel, are not necessary for the differentiation and patterning of the organ of Corti. In addition, the results of a number of studies have suggested potential roles for soluble growth factors, such as retinoic acid and thyroid hormones in the differentiation of hair cells (Raz and Kelley, 1999; Rusch et al., 2001). Although these results do not refute those findings, they do suggest that most of these growth factors are probably not derived from distant sources and that it is more likely that any growth factors that are required for cellular differentiation and patterning are probably produced by other cells within the epithelium.

\section{Planar signals originating in the mid-basal region of the cochlea can regulate cellular differentiation and patterning along the length of the cochlea}

The morphological gradient of differentiation that initiates in the mid-basal region of the cochlea and progresses toward both the apex and the base was initially described by Kolliker (1861) and Retzius (1884). Since that time, these observations have been confirmed at both morphological and molecular levels (for review, see Kelley and Bianchi, 2001). The presence of this gradient suggested that the signals required for the differentiation of the sensory epithelium originate in the mid-basal region of the cochlear duct and then progress through the plane of the epithelium over time; however, an alternative possibility is that the signals required for differentiation are initially present throughout the epithelium, or are derived from another source, and that the observed gradient in development is reflective of a gradient in the timing of the onset of differentiation. To examine whether developmental signals that are required for cellular differentiation and patterning progress within the plane of the developing cochlear epithelium, the differentiation of hair cells and the development of cellular pattern were compared in isolated apical cochlear epithelial pieces and comparable apical regions in intact cochlear epithelia from E12.5 + 6 DIV/-mes explants. In intact epithelia, hair cells developed along the entire length of the explant, including the apical region. In contrast, no hair cells developed in the majority of isolated apical explants, and in those explants with hair cells, the average number was fewer than eight per explant (compare the apical region in Fig. $2 C$ with Fig. $4 B$ ). These results strongly suggest that signals required for hair cell differentiation are conveyed within the plane of the epithelium along the basal-to-apical axis of the developing cochlea and that these signals are sufficient to induce hair cell differentiation, even in the absence of mesenchymal signals. Moreover, the observation that cellular patterning is disrupted in apical pieces from E13.5 + 5 DIV/ - mes, although hair cell differentiation does occur, suggests the possibility that discreet signals are required for differentiation and patterning.

The nature of this signal or signals is unclear. The overall length of the cochlea at E12.5 $(\sim 4.5 \mathrm{~mm})$ and its coiled morphology might argue against a soluble factor; however, if the effective distance of this signal were short (one to five cell diameters), then the relatively broad arc of the cochlear spiral might not significantly affect the shape of the diffusion gradient. Alternatively, the instructive signal could be mediated through cell-cell contacts. Finally, a comparison with the signals that control the propagation of the morphogenetic furrow that patterns the eye imaginal disk in Drosophila (for review, see Reifegerste and Moses, 1999; Brennan and Moses, 2000) would suggest that an initial diffusible signal might be subsequently reinforced through local cell-cell interactions.

Specific vertical cues from adjacent mesenchyme can regulate hair cell differentiation and patterning

Previous in vitro studies had demonstrated an important role for associated mesenchymal cells in the early development of the 
otocyst (Van de Water, 1983; Anniko and Schacht, 1984; Anniko, 1985). In the study by Anniko and Schacht (1984), however, the disruptive effects of removing the mesenchyme could be rescued if otocyts were maintained in media conditioned by inner ear mesenchymal cells or mesenchymal cells from other regions of the head or body, suggesting a nonspecific effect. In contrast, the results presented here strongly suggest that mesenchymal cells associated with the cochlear duct produce signals that are specific for the differentiation and patterning of the organ of Corti. One possibility is that a diffusible factor could originate in the relatively small number of mesenchymal cells that are located adjacent to the developing organ of Corti. There are presently no data to support this hypothesis; however, preliminary results suggest that the mesenchymal cells located directly adjacent to the developing organ of Corti may be distinct, at a molecular level, from the mesenchymal cells that surround the rest of the cochlea duct (M. W. Kelley and K. B. Avraham, unpublished data). Although the nature of these signals is not known, the results of the conditioned media and coculture experiments presented here suggest that these signals are mediated through cell-cell contact, shortrange diffusible signals, and/or components of the extracellular matrix (Davies and Holley, 2002).

\section{Both planar and vertical cues can regulate the development of the organ of Corti}

The results presented here demonstrate that hair cell differentiation in the apical region of the developing cochlea at E12.5 is dependent on signals arising either from a more basal region of the cochlear epithelium or from associated mesenchymal cells. If both of these structures are removed on E12.5, then hair cell differentiation is significantly disrupted in the apical epithelium; however, association with either structure is sufficient to induce hair cell differentiation. These results suggest that these two signaling sources are complementary. On the basis of these results, it seems reasonable to suggest that functional parallels may exist between the mechanisms that play a role in the development of the cochlea and the mechanisms that regulate primary neurogenesis in vertebrates. In both cases, cues arise from two sources: a planar source that is present within the epithelium and a vertical source that arises from adjacent mesodermally derived cells (for review, see Weinstein and Hemmati-Brivanlou, 1999; Wilson and Edlund, 2001).

\section{Timing of cellular differentiation in the cochlea}

The observation that hair cell differentiation was not disrupted in E13.5 + 5 DIV apical epithelia suggested that planar signals have already progressed into the apex by this time. Consistent with this hypothesis, a single row of myosin VI-positive cells was observed to extend along the basal-most $75 \%$ of the basal-to-apical axis of the cochlea at E13.5. These cells presumably represent the initial population of differentiating inner hair cells. The presence of an initial population of myosin VI-positive hair cells at E13.5 is also consistent with previous reports of Math1 expression in the cochlea between E12.5 and E13 (Bermingham et al., 1999; Lanford et al., 1999).

These results differ with recent results suggesting that Math1, a transcription factor that is both necessary and sufficient for hair cell differentiation (Bermingham et al., 1999), is not expressed in the cochlea before E14.5 (Chen et al., 2002). A possible explanation for this discrepancy is that the level of Math1 expression in this early population of hair cells at E13.5 was too low to be detected using the methods used by Chen et al. (2002).

\section{Timing of cellular differentiation is not regulated by planar signals}

In the mammalian cochlea there is an apparent dichotomy between the apical-to-basal gradient of terminal mitoses (Ruben, 1967) and the subsequent basal-to-apical gradient of cellular differentiation (for review, see Kelley and Bianchi, 2001). Because of these counter-gradients, the progenitor cells located in the apicalmost region of the cochlea must remain in a postmitotic, undifferentiated state for at least several days in the mouse and possibly for even longer periods in some other mammals, including humans. On the basis of these observations, one possible role of planar signals could be to regulate the timing of cellular differentiation by maintaining apical progenitor cells in an undifferentiated state; however, the timing of cellular differentiation was not affected in apical pieces that had been separated from the base at E12.5. These results suggest that although planar signals play a role in hair cell development and patterning, the timing of cellular differentiation appears to be either intrinsic to the progenitor cells or specified through signals that are present in the apical portion of the epithelium before E12.5.

In summary, the results presented here demonstrate that signals contained within the plane of the developing cochlear epithelium provide cues that regulate cellular differentiation and patterning. In addition, mesenchymal cells located adjacent to developing cochlear epithelial cells apparently produce vertical signals that can also influence cochlear development. These two signals appear to complement one another, such that the loss of one signal can be compensated for by the other signal. Moreover, the results demonstrate a new role for the mesenchymal cells that develop in close association with the cochlear duct.

\section{References}

Abdouh A, Despres G, Romand R (1994) Histochemical and scanning electron microscopic studies of supernumerary hair cells in embryonic rat cochlea in vitro. Brain Res 660:181-191.

Anniko M (1985) Hair cell differentiation following tissue interactions for induction of otocyst morphogenesis. Arch Otorhinol 242:287-294.

Anniko M, Schacht J (1984) Inductive tissue interactions during inner ear development. Arch Otorhinolaryngol 240:17-33.

Avraham KB, Hasson T, Steel KP, Kingsley DM, Russell LB, Mooseker MS, Copeland NG, Jenkins NA (1995) The mouse Snell's waltzer deafness gene encodes an unconventional myosin required for structural integrity of inner ear hair cells. Nat Genet 11:369-375.

Bermingham NA, Hassan BA, Price SD, Vollrath MA, Ben-Arie N, Eatock RA, Bellen HJ, Lysakowski A, Zoghbi HY (1999) Math1: an essential gene for the generation of inner ear hair cells. Science 284:1837-1841.

Brennan CA, Moses K (2000) Determination of Drosophila photoreceptors: timing is everything. Cell Mol Life Sci 57:195-214.

Chen P, Segil N (1999) p27(Kip1) links cell proliferation to morphogenesis in the developing organ of Corti. Development 126:1581-1590.

Chen P, Johnson JE, Zoghbi HY, Segil N (2002) The role of Math1 in inner ear development: uncoupling the establishment of the sensory primordium from hair cell fate determination. Development 129:2495-2505.

Colvin JS, Bohne BA, Harding GW, McEwen DG, Ornitz DM (1996) Skeletal overgrowth and deafness in mice lacking fibroblast growth factor receptor 3. Nat Genet 12:390-397.

Davies D, Holley MC (2002) Differential expression of alpha 3 and alpha 6 integrins in the developing mouse inner ear. J Comp Neurol 445:122-132.

Fuhrmann S, Levine EM, Reh TA (2000) Extraocular mesenchyme patterns the optic vesicle during early eye development in the embryonic chick. Development 127:4599-4609.

Gale JE, Marcotti W, Kennedy HJ, Kros CJ, Richardson GP (2001) FM1-43 dye behaves as a permeant blocker of the hair-cell mechanotransducer channel. J Neurosci 21:7013-7025.

Kaufman MH (1992) The atlas of mouse development. San Diego: Academic.

Kelley MW, Bianchi LM (2001) Development and neuronal innervation of the organ of Corti. In: Handbook of the mouse auditory research: from 
behavior to molecular biology (Willott JF, ed), pp 137-156. New York: CRC.

Kelly OG, Melton DA (1995) Induction and patterning of the vertebrate nervous system. Trends Genet 11:273-278.

Lanford PJ, Lan Y, Jiang R, Lindsell C, Weinmaster G, Gridley T, Kelley MW (1999) Notch signaling pathway mediates hair cell development in mammalian cochlea. Nat Genet 21:289-292.

Lanford PJ, Shailam R, Norton CR, Gridley T, Kelley MW (2000) Expression of Math1 and HES5 in the cochleae of wildtype and Jag2 mutant mice. J Assoc Res Otolaryngol 1:161-171.

Lavigne-Rebillard M, Pujol R (1987) Surface aspects of the developing human organ of Corti. Acta Otolaryngol [Suppl] 436:43-50.

Lim DJ, Anniko M (1985) Developmental morphology of the mouse inner ear. Acta Otolaryngol [Suppl] 422:1-69.

Liu W, Oh SH, Kang YK, Li G, Doan TM, Little M, Li L, Ahn K, Crenshaw III EB, Frenz DA (2003) Bone morphogenetic protein 4 (BMP4): a regulator of capsule chondrogenesis in the developing mouse inner ear. Dev Dyn 226:427-438.

Malbouisson JM, Baccega A, Cruz AA (2000) The geometrical basis of the eyelid contour. Ophthalmol Plast Reconstr Surg 16:427-431.

Mansour SL, Goddard JM, Capecchi MR (1993) Mice homozygous for a targeted disruption of the proto-oncogene int- 2 have developmental defects in the tail and inner ear. Development 117:13-28.

Maroon H, Walshe J, Mahmood R, Kiefer P, Dickson C, Mason I (2002) Fgf3 and Fgf8 are required together for formation of the otic placode and vesicle. Development 129:2099-2108.

Matsui JI, Ogilvie JM, Warchol ME (2002) Inhibition of caspases prevents ototoxic and ongoing hair cell death. J Neurosci 22:1218-1227.

Meyers JR, MacDonald RB, Duggan A, Lenzi D, Standaert DG, Corwin JT, Corey DP (2003) Lighting up the senses: FM1-43 loading of sensory cells through nonselective ion channels. J Neurosci 23:4054-4065.

Montcouquiol M, Corwin JT (2001) Intracellular signals that control cell proliferation in mammalian balance epithelia: key roles for phosphatidylinositol-3 kinase, mammalian target of rapamycin, and S6 kinases in preference to calcium, protein kinase $\mathrm{C}$, and mitogen-activated protein kinase. J Neurosci 21:570-580.

Montcouquiol M, Rachel RA, Lanford PJ, Copeland NG, Jenkins NA, Kelley MW (2003) Identification of Vangl2 and Scrb1 as planar polarity genes in mammals. Nature 423:173-177.
Raz Y, Kelley MW (1999) Retinoic acid signaling is necessary for the development of the organ of Corti. Dev Biol 213:180-193.

Reifegerste R, Moses K (1999) Genetics of epithelial polarity and pattern in the Drosophila retina. BioEssays 21:275-285.

Retzius G (1884) Das Gehororgan der Wirbeltiere. II. Das Gehororgan der Reptilien, der Vogel und Saugetiere. Stockholm: Samson and Wallin.

Ruben RJ (1967) Development of the inner ear of the mouse: a radioautographic study of terminal mitoses. Acta Otolaryngol 220:1-44.

Rusch A, Ng L, Goodyear R, Oliver D, Lisoukov I, Vennstrom B, Richardson G, Kelley MW, Forrest D (2001) Retardation of cochlear maturation and impaired hair cell function caused by deletion of all known thyroid hormone receptors. J Neurosci 21:9792-9800.

Sher AE (1971) The embryonic and postnatal development of the inner ear of the mouse. Acta Otolaryngol [Suppl] 285:1-77.

Van de Water TR (1983) Embryogenesis of the inner ear: in vitro studies. In: Development of auditory and vestibular systems (Romand R, ed), pp 337-372. New York: Academic.

van Ruijven LJ, Beek M, van Eijden TM (1999) Fitting parametrized polynomials with scattered surface data. J Biomech 32:715-720.

Weil D, Blanchard S, Kaplan J, Guilford P, Gibson F, Walsh J, Mburu P, Varela A, Levilliers J, Weston MD, Kelley PM, Kimberling WJ, Wagenaar M, Levi-Acobas F, Larget-Piet D, Munnich A, Steel KP, Brown SDM, Petit C (1995) Defective myosin VIIA gene responsible for Usher syndrome type 1B. Nature 374:60-61.

Weinstein DC, Hemmati-Brivanlou A (1999) Neural induction. Annu Rev Cell Dev Biol 15:411-433.

Wessely O, De Robertis EM (2002) Neural plate patterning by secreted signals. Neuron 33:489-491.

Wilson SL, Edlund T (2001) Neural induction: toward a unifying mechanism. Nat Neurosci 1:1161-1168.

Wright TJ, Mansour SL (2003) Fgf3 and Fgf10 are required for mouse otic placode induction. Development 130:3379-3390.

Xiang M, Gao WQ, Hasson T, Shin JJ (1998) Requirement for Brn-3c in maturation and survival, but not in fate determination of inner ear hair cells. Development 125:3935-3346.

Zheng J, Shen W, He DZ, Long KB, Madison LD, Dallos P (2000) Prestin is the motor protein of cochlear outer hair cells. Nature 405:149-155. 